\title{
High-quality multi-GeV electron bunches via cyclotron autoresonance
}

\author{
Benjamin J. Galow, ${ }^{1}$ Jian-Xing Li, ${ }^{1}$ Yousef I. Salamin,,${ }^{1,2}$ Zoltán Harman,,${ }^{1,3}$ and Christoph H. Keitel ${ }^{1}$ \\ ${ }^{1}$ Max-Planck-Institut für Kernphysik, Saupfercheckweg 1, 69029 Heidelberg, Germany \\ ${ }^{2}$ Department of Physics, American University of Sharjah, POB 26666, Sharjah, United Arab Emirates \\ ${ }^{3}$ ExtreMe Matter Institute EMMI, Planckstrasse 1, 64291 Darmstadt, Germany
}

(Received 2 October 2012; published 15 August 2013)

\begin{abstract}
Autoresonance laser acceleration of electrons is theoretically investigated using circularly polarized focused Gaussian pulses. Many-particle simulations demonstrate feasibility of creating over 10-GeV electron bunches of ultrahigh quality (relative energy spread of order $10^{-4}$ ), suitable for fundamental high-energy particle physics research. The laser peak intensities and axial magnetic field strengths required are up to about $10^{18} \mathrm{~W} / \mathrm{cm}^{2}$ (peak power $\sim 10 \mathrm{PW}$ ) and $60 \mathrm{~T}$, respectively. Gains exceeding $100 \mathrm{GeV}$ are shown to be possible when weakly focused pulses from a 200-PW laser facility are used. In our parametric study of this acceleration scheme, substantial challenges still need to be dealt with, especially on the road to realizing experimentally the required high magnetic field strengths and laser powers.
\end{abstract}

DOI: 10.1103/PhysRevSTAB.16.081302

PACS numbers: 52.38.Kd, 37.10.Vz, 42.65.-k, 52.75.Di

\section{INTRODUCTION}

Particle accelerators are an indispensable tool to explore the fundamental laws of nature and are widely used for medical and industrial applications. At the frontier of accelerator technology is the Large Hadron Collider (LHC), a gigantic circular machine of $27 \mathrm{~km}$ total circumference [1]. The need to control the size and cost of building such machines have kept alive the quest for alternative means to accelerate particles. Over the past decade, laser plasma-based acceleration has emerged as a promising candidate [2-8]. In particular, laser wakefield acceleration of electrons [9-11] has undergone rapid development. Stable and reproducible beams have been realized [12] and particle kinetic energies at the GeV level have been reached [13]. Furthermore, the creation of a plasma wave from interaction with a highly energetic electron beam as a driver allows for doubling the kinetic energy of the accelerated particles within a meter-scale plasma wakefield accelerator [14-16].

The advent of quasistatic magnetic fields [17-21] of durations up to seconds, with strengths as high as 100 Tesla, suggests vacuum autoresonance laser acceleration (ALA) (see [22,23] and references therein) as a further potential alternative to conventional acceleration. The ALA mechanism employs a static magnetic field oriented along the propagation direction of the laser. Thus, the underlying concept of ALA stems from the realization that an electron continues to absorb energy from a circularly polarized laser field if it is launched in cyclotron

Published by the American Physical Society under the terms of the Creative Commons Attribution 3.0 License. Further distribution of this work must maintain attribution to the author(s) and the published article's title, journal citation, and DOI. autoresonance with it. For the case of laser fields described by plane waves [23] resonance is essentially between the Doppler-shifted laser frequency seen by the electron and the cyclotron frequency of the electron around the lines of the applied static magnetic field. Feasibility of postacceleration of electrons to kinetic energies of about 3 times their initial energies has also been theoretically investigated, employing continuous-wave $\mathrm{CO}_{2}$ laser fields described within the paraxial approximation [24].

In this paper, the ALA configuration (see Fig. 1) is investigated over a wide range of laser and magnetic field parameters. Our results, stemming from single- and manyparticle calculations which employ pulsed and focused laser fields, indicate electron energy gains of several hundred (even thousand) times the initial injection energy. For magnetic field strengths below 60 Tesla, energy gains in excess of $10 \mathrm{GeV}$ are shown to be possible. It is shown that the gains are attained over distances less than $10 \mathrm{~m}$,

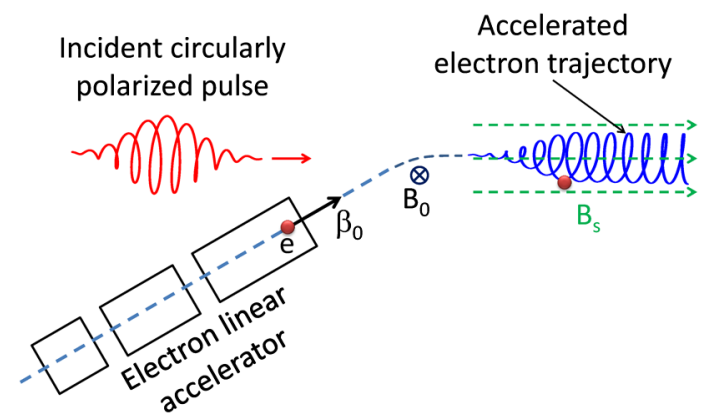

FIG. 1. A schematic showing the vacuum ALA configuration. A linear accelerator (linac) preaccelerates the electron, labeled by $e$ and the velocity vector $\boldsymbol{\beta}_{0}$, to $50 \mathrm{MeV}$ and a magnetic field $B_{0}$ bends its trajectory slightly for injection axial to the combination of incident laser pulse and axial uniform magnetic field of strength $B_{s}$. 
and from a near-infrared laser system of peak intensity $<10^{18} \mathrm{~W} / \mathrm{cm}^{2}$ (peak power $\sim 10 \mathrm{PW}$ ). Many-particle simulations also demonstrate that an electron bunch of high quality (relative energy spread on the $10^{-4}$ level) may be obtained, taking Coulomb particle-particle repulsions into account in simulations at densities of $10^{15} \mathrm{~cm}^{-3}$. Gains in excess of $100 \mathrm{GeV}$ are also shown to be possible anticipating a 200-PW laser system, like it might be realized by the extreme light infrastructure (ELI) [25]. Our work is motivated by currently feasible magnetic fields of strength of the order of 100 Tesla $[17,18]$ and anticipates continued progress in high magnetic field research.

\section{BASIC EQUATIONS}

Classical electron motion in the presence of electromagnetic fields $\boldsymbol{E}$ and $\boldsymbol{B}$ is governed by the Lorentz-Newton equations of motion, namely,

$$
\frac{d \boldsymbol{p}}{d t}=-e(\boldsymbol{E}+c \boldsymbol{\beta} \times \boldsymbol{B}) \quad \text { and } \quad \frac{d \mathcal{E}}{d t}=-e c \boldsymbol{\beta} \cdot \boldsymbol{E},
$$

which describe time evolution of the particle's relativistic momentum $\boldsymbol{p}=\gamma m c \boldsymbol{\beta}$ and energy $\mathcal{E}=\gamma m c^{2}$, respectively. In the above, $e$ is the magnitude of the charge of the electron and $m$ is its rest mass, $c$ is the speed of light in vacuum, $\boldsymbol{\beta}$ is the particle's velocity scaled by $c, \gamma=$ $\left(1-\beta^{2}\right)^{-1 / 2}$ and SI units are used throughout. Results to be presented below are based on solving these equations numerically for single- and many-particle systems. In the solutions, the electron is assumed to be overcome by the front of the pulse at $t=0$ at the origin of a coordinate system whose $z$ axis is oriented along the direction of pulse propagation.

In earlier calculations of autoresonance acceleration the laser fields were modeled as plane waves of infinite extension in space and time [23], or as those of a continuous beam within the paraxial approximation [24]. The planewave-based calculations have led to the realization of the resonance condition, to be recalled below, and have shown that an electron stands to gain more energy from circularly polarized light than from light of the linear polarization variety. Recall that the polarization vector of a circularly polarized plane wave rotates about the direction of propagation at the angular frequency of the wave, while its field strength remains constant. Thus, if the initial conditions are such that the electron cyclotron frequency matches the Doppler-shifted frequency (sensed by the electron) of the circularly polarized fields, the electron will subsequently surf on the wave and continue to absorb energy from it. Hence, calculations in this paper will employ circularly polarized laser fields, obtained by the superposition of two linearly polarized fields, with perpendicular polarization vectors and a $\pi / 2$-phase difference [26]. A pulse shape is introduced by multiplying the fields by the Gaussian envelope $\exp \left(-\eta^{2} / 2 \sigma^{2}\right)$, where $\eta=\omega t-k z$ is the phase variable, $k=2 \pi / \lambda$ is the wave number, $\sigma=\omega \tau /(2 \sqrt{2 \ln 2})$ is the envelope's full-width-at-half-maximum, and $\tau$ is the pulse duration (temporal full-width-at-half-maximum). For the field amplitudes, a generalized Lax series representation (in powers of the diffraction angle $\epsilon=\lambda / \pi w_{0}$, where $w_{0}$ is the beam's waist radius at focus) will be adopted [26-28]. Thus, the fields of the ALA scheme may be written as (see [26] for definitions of the symbols and more details)

$$
\boldsymbol{E}=e^{-\eta^{2} / 2 \sigma^{2}}\left\{\left[E_{x} \hat{\boldsymbol{e}}_{x}+E_{x}^{\prime} \hat{\boldsymbol{e}}_{y}\right]+\left[E_{y} \hat{\boldsymbol{e}}_{y}+E_{y}^{\prime} \hat{\boldsymbol{e}}_{x}\right]+\left[E_{z}+E_{z}^{\prime}\right] \hat{\boldsymbol{e}}_{z}\right\},
$$

and

$$
\boldsymbol{B}=e^{-\eta^{2} / 2 \sigma^{2}}\left\{\left[B_{y} \hat{\boldsymbol{e}}_{y}-B_{y}^{\prime} \hat{\boldsymbol{e}}_{x}\right]+\left[B_{z}-B_{z}^{\prime}\right] \hat{\boldsymbol{e}}_{z}\right\}+B_{s} \hat{\boldsymbol{e}}_{z},
$$

where the primed components follow from the unprimed ones by letting $x \leftrightarrow y$ and adding a phase shift of $-\pi / 2$.

\section{THE FIELDS}

To decide the order of the correction terms, beyond the paraxial approximation, which ought to be retained in the various field expressions, simulations have been performed for a single electron injected axially with $50 \mathrm{MeV}$ initial kinetic energy. The electron's exit energy gain as a function of the pulse waist radius at focus has been analyzed, when terms up to $\mathcal{O}\left(\epsilon^{n}\right)$, where $n=0,1,2,3$, are employed in modeling the laser fields (see Fig. 2). The simulation results for terms of highest order $\epsilon^{2}$ and $\epsilon^{3}$ coincide, demonstrating that terms of order higher than $\epsilon^{2}$ may be dropped, as expected, since $\epsilon \ll 1$.

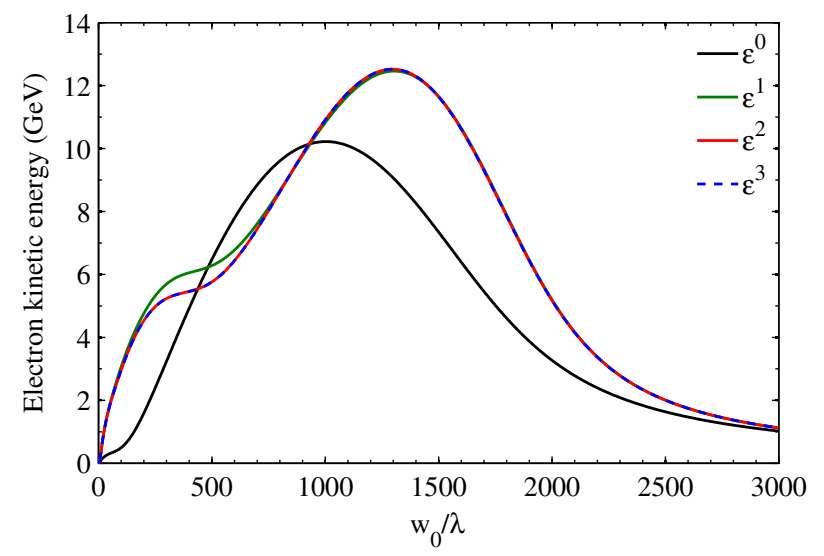

FIG. 2. Electron exit energy gain as a function of the laser beam radius at focus, employing field representations of different orders in the diffraction angle. The electron is injected along the $z$ axis with $50 \mathrm{MeV}$ initial kinetic energy and initial location at the origin of coordinates $x=y=z=0$. The laser system parameters are $\lambda=1 \mu \mathrm{m}$, power $P=10 \mathrm{PW}$, and pulse duration $\tau=25 \mathrm{fs}$. The trajectory and the excursion distance of the electron for the set of parameters leading to maximal kinetic energy gain are shown in Fig. 4(a). 


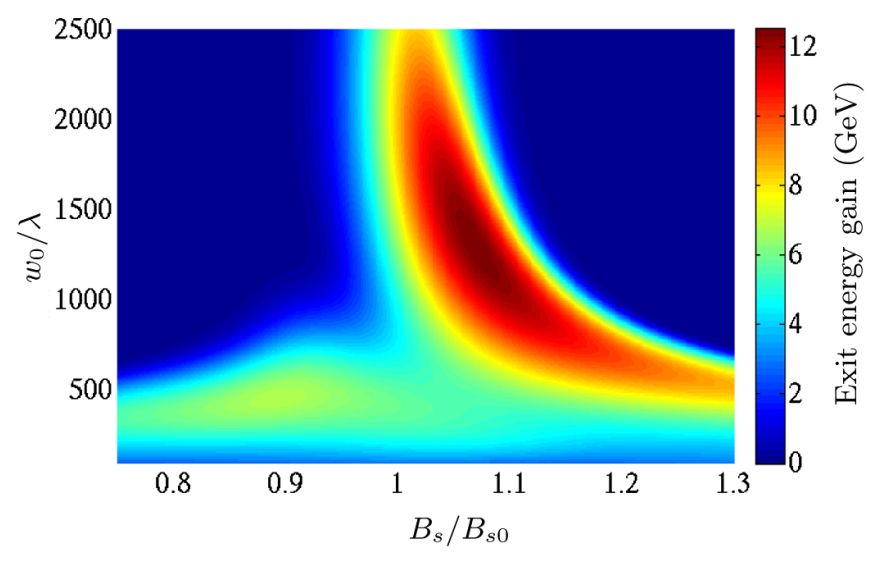

FIG. 3. Contour plot of the exit energy gain as a function of the beam radius at focus $w_{0}$ and the external static magnetic field $B_{s}$. The laser and electron injection parameters are the same as in Fig. 2.

Because of the large focus radius, the longitudinal component influences the ALA dynamics only negligibly and the energy gain results essentially from interaction with the transverse field component. This is shown in analytical calculations in [23] for purely transverse fields. For the parameters used (see caption of Fig. 3), the exit energy gain peaks for a waist radius at focus $w_{0} \sim 1295 \lambda$. The maximum electron exit energy gain attained in this case is $K \sim 12.5 \mathrm{GeV}$. The peak is reached for a focus large enough to allow for optimal autoresonance to occur, but still tight enough to guarantee a sufficiently strong field $\left(E_{0} \propto 1 / w_{0}\right)$.

\section{CYCLOTRON AUTORESONANCE}

From the plane-wave calculations [23] we learn that, for autoresonance to occur, the uniform magnetic field, to be applied axially and added to the laser magnetic field in the equations of motion, should be calculated from

$$
B_{s 0}=\frac{m \omega}{e} \sqrt{\frac{1-\beta_{0}}{1+\beta_{0}}},
$$

where $\omega$ is the laser frequency, and $\beta_{0}$ is the initial speed of the electron scaled by $c$. Introduction of a pulse shape and a tight focus is expected to modify the resonance condition (4) and render it approximate at best, thus leading to a slight deterioration in the electron-beam quality. To investigate this issue, the applied axial magnetic field $B_{s 0}$ is replaced by $B_{s}$ and the parameter space, spanned by $w_{0} / \lambda$ and $B_{s} / B_{s 0}$, is scanned for optimum exit energy gains. The results are displayed in Fig. 3; a contour plot of the exit energy gain vs both the beam waist radius at focus $w_{0}$ and the employed static magnetic field $B_{s}$. The plot shows clearly a region in parameter space for which the exit energy gain is optimal. The exit energy gain is not sensitive to small fluctuations in the ALA configuration parameters. For example, an energy gain of about $11 \mathrm{GeV}$ may be realized for waist radii $w_{0}$ in the range extending roughly from $950 \lambda$ to $1750 \lambda$ and a magnetic field strength $B_{s}$ in the approximate range $1.04 B_{s 0}$ to $1.13 B_{s 0}$. The maximum energy gain of $12.5 \mathrm{GeV}$ is reached for $B_{s}=1.07 B_{s 0} \sim$ $58 \mathrm{~T}$. Recent progress in quasistatic magnetic field research has achieved $45 \mathrm{~T}[18,20,21]$ over a distance of $0.225 \mathrm{~m}$. Thus, the $58 \mathrm{~T}$ goal may not be too far fetched, and the need to have such a magnetic field strength over $25 \mathrm{~m}$ may, in principle, be met by employing an assembly of such magnets. However, such a stacking of magnets will be experimentally difficult to realize and the generated fringe fields at the interface between two magnets will probably have negative impact on the energy resolution.

To continuously maintain a magnetic field strength of about $60 \mathrm{~T}$ over $10 \mathrm{~m}$ for one second would require an average power consumption of $2.25 \mathrm{GW}$ [18]. However, a petawatt laser provides its energy in a pulsed way, such that we do not need to continuously maintain the magnetic field which will significantly lower the average energy consumption as we will show subsequently. For one relativistic electron bunch at approximately the speed of light it takes about $33.3 \mathrm{~ns}$ to travel $10 \mathrm{~m}$ and, hence, for ten bunches about $0.3 \mu \mathrm{s}$. This implies that at a laser repetition rate of ten shots per second the energy consumption of the ALA scheme corresponds to only $0.7 \mathrm{~kJ}$ based on pulsed magnets. Since this value does not include the power consumption of the laser system and the linac, it exceeds in total the power consumption of laser wakefield accelerators $[29,30]$. Moreover, we want to emphasize that this estimate is based on the assumptions that the magnets are able to operate at such short pulses and their power consumption scales linearly with the magnets' pulse duration, which might be influenced by the presence of pedestals.

\section{ALA DYNAMICS}

\section{A. Single-particle calculations}

For further insight into the ALA dynamics, Fig. 4(a) shows the 3D trajectory of a single electron, accelerated using the optimal parameters of $w_{0}=1295 \lambda$ and $B_{s}=$ $1.07 B_{s 0}$. Note that the transverse dimensions are enlarged in Fig. 4(a) giving the impression that the trajectory is a helix with increasing radius. However, the radius of the helix hardly increases beyond the first $9 \mathrm{~m}$ of axial excursion, over which the pitch of the helix increases rapidly, thus rendering the trajectory essentially linear. In Fig. 4(b) the corresponding exit energy gain (red dashed line) is shown as a function of the axial excursion distance. After an initial (approximately linear) increase, the energy gain starts to saturate with increasing excursion. The accelerating phase seems to be limited within the initial axial excursion, after which interaction with the transverse electric field components diminishes as the particle gets left behind the pulse. For the set of parameters used (see caption of Fig. 3), the $10-\mathrm{GeV}$ level is reached after a 

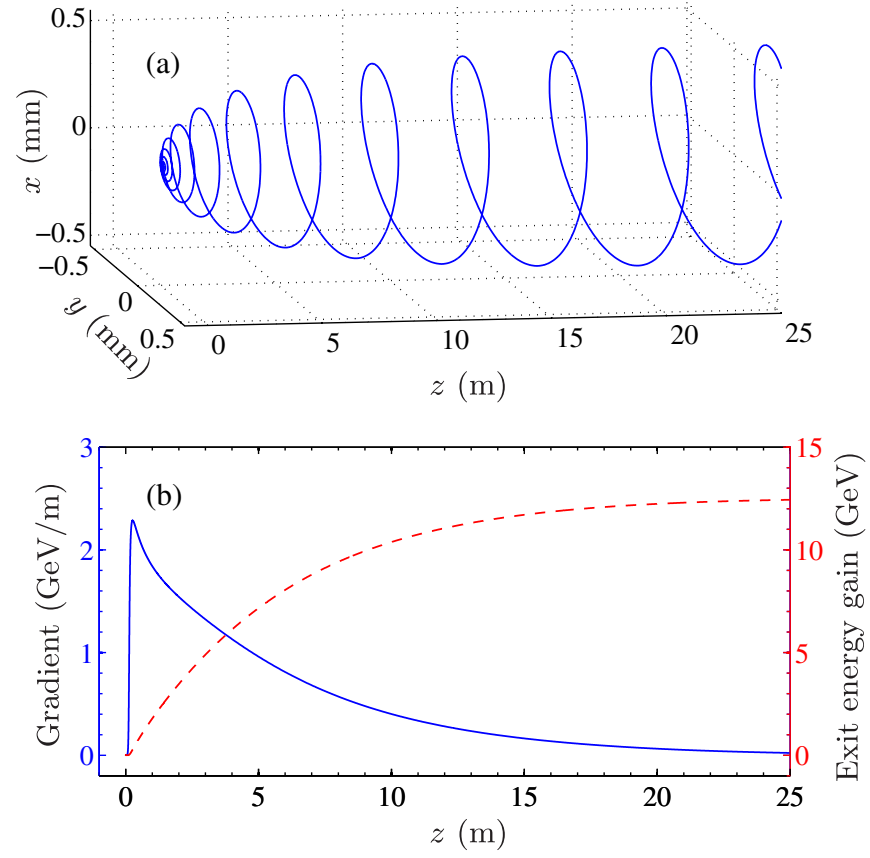

FIG. 4. (a) 3D trajectory of a single electron in autoresonance interaction with laser and static magnetic fields, for $w_{0} \sim 1295 \lambda$ and $B_{s}=1.07 B_{s 0}$. (b) Acceleration gradient (blue line, left ordinate) and exit energy gain (red dashed line, right ordinate) vs the axial excursion distance for the same electron. The laser peak intensity is $I_{0} \sim 7.59 \times 10^{17} \mathrm{~W} / \mathrm{cm}^{2}$, and other parameters are the same as in Fig. 3.

9.14-m axial excursion. Moreover, the acceleration gradient $d G / d z=-e \boldsymbol{\beta} \cdot \boldsymbol{E} / \beta_{z}$ is plotted vs the axial excursion $z$ (blue line). The maximal acceleration gradient of $2.286 \mathrm{GeV} / \mathrm{m}$ is reached at $z=0.2626 \mathrm{~m}$. This exceeds the gradient of conventional linacs $(100 \mathrm{MeV} / \mathrm{m})$ by more than 1 order of magnitude. By contrast, wakefield accelerators reach gradients of $10-100 \mathrm{GeV} / \mathrm{m}$ [13]. However, it should be emphasized that in single-stage wakefield accelerators the acceleration distance is typically $<1 \mathrm{~m}$, yielding energy spreads of the order of $2 \%$ [10].

\section{B. Many-particle simulations}

The single-particle calculations, whose results have been presented above, will now be supported by manyparticle simulations. Dynamics of a bunch of electrons injected along the $z$ axis into the ALA configuration, as well as the beam properties of the accelerated electrons, are considered next. An ensemble of electrons, considered to be noninteracting for now, randomly distributed within a volume of cylindrical shape centered about the coordinate origin and oriented along the $z$ axis, is used to model an electron bunch, along the lines of our earlier work in [31-33]. The incident laser pulse accelerates particles at the left end of the cylinder first, followed by particles farther to the right. The cylinder containing the electrons has a length $l_{c}=1 \mathrm{~mm}$ and a radius $r_{c}=0.05 \mathrm{~mm}$.
The initial kinetic energy of the electrons follows a normal distribution with mean value $K_{0}=50 \mathrm{MeV}$ and spread (standard deviation) $\Delta K_{0}=0.05 \mathrm{MeV}$ [34]. Such an electron bunch may be preaccelerated using a short linac or a tabletop betatron, and then guided by a magnetic field for axial injection (see Fig. 1). Employing the laser system parameters of Fig. 3 and the optimal waist radius $w_{0}=$ $1295 \lambda$, the resulting exit energy gain distribution of an ensemble of 15000 electrons has a mean exit energy gain of $G_{\text {exit }}=12.513 \mathrm{GeV}$ and a spread of $\Delta G_{\text {exit }}=3.7 \mathrm{MeV}$ $(0.0293 \%)$. The transverse beam emittance amounts to $\approx 0.1 \pi \mathrm{mmmrad}$ which compares well with what is obtained from conventional accelerators [34].

Dependence of the electron exit kinetic energy distribution on fluctuations in the initial kinetic energy distribution has been studied. Employing a bunch with $K_{0}=50 \pm 0.5 \mathrm{MeV}$ changes the mean exit energy gain to $G_{\text {exit }}=12.296 \mathrm{GeV}$, and its spread to $\Delta G_{\text {exit }}=$ $0.321 \mathrm{GeV}(2.61 \%)$.

\section{Particle-particle interaction effects}

To investigate the role of electron-electron interaction effects a suitable model has to be developed. A conventional particle-in-cell scheme describes the interaction of a laser with an initially neutral plasma, and is not applicable over macroscopic distances of several meters. Therefore, further simulations have been performed employing a 1000particle ensemble confined to a spatial volume similar to what has been used above, but scaled to render the particle density the same as would be obtained from a $10^{10}$-particle bunch (typical in conventional particle accelerators [35]) with the Coulomb interactions turned on [36] and off. The resulting exit energy gain distributions are shown in Fig. 5. In the noninteracting ensemble case, Fig. 4(a), the mean exit energy gain amounts to $G_{\text {exit }}=12.518 \mathrm{GeV}$ with a spread of $\Delta G_{\text {exit }}=3.17 \mathrm{MeV}(0.0253 \%)$. For the interacting ensemble, Fig. 4(b), the energy spread approximately doubles $\left[G_{\text {exit, } 1000}^{\text {Coulo }}=12.519 \mathrm{GeV}, \Delta G_{\text {exit, } 1000}^{\text {Comb }}=6.22 \mathrm{MeV}\right.$ $(0.0497 \%)]$. Furthermore, spatial spreading of the bunch (not shown here) is increased by a few percent as a result.
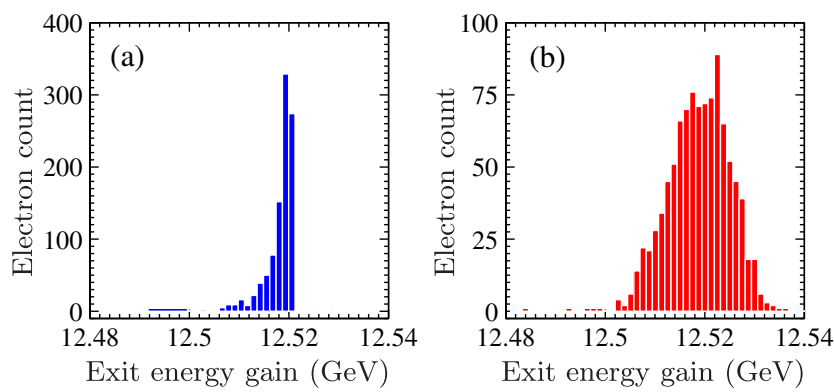

FIG. 5. Distribution of the exit energy gain amongst 1000 (a) noninteracting and (b) interacting electrons in an ALA scheme. See Figs. 3 and 4 for the laser and injection parameters. 
Since the relative velocities of electrons in the center of mass of the bunch are low $\left(\beta_{\text {rel. }} \lesssim 10^{-3}\right)$, higher-order relativistic particle-particle interaction effects [36] can be neglected. To ensure that the reduced size of the ensemble does not play a role, we performed the simulations for an interacting ensemble of 500 particles at the same density. Bearing in mind the different random initial conditions, a mean exit energy gain of $G_{\text {exit, } 500}^{\text {Comb }}=12.519 \mathrm{GeV}$ with a spread of $\Delta G_{\text {exit } 500}^{\text {Coulomb }}=5.18 \mathrm{MeV}(0.0420 \%)$ has been obtained, which is in good agreement with the values given above for the 1000-particle ensemble. Hence, validity of the calculational method is confirmed and the long-range interaction effects have been treated appropriately.

\section{DISCUSSION}

The examples discussed thus far have been concerned essentially with a single set of laser parameters, soon to be available for laboratory experiments. Even more powerful laser systems may be available in the near future [25]. In search of parameter sets that may lead to much higher energy gains, useful for particle physics research, singleparticle calculations have been performed whose results are displayed in Fig. 6. The figure shows, e.g., that a gain of about $100 \mathrm{GeV}$ may be reached employing a 75 -fs pulse, focused to $w_{0}=6800 \lambda$ and derived from a 200-PW laser system (corresponding to a peak intensity $I_{0} \sim 4.41 \times$ $10^{17} \mathrm{~W} / \mathrm{cm}^{2}$ ) [25]. However, this will come at a price to be paid in terms of the size of such a facility, and a uniform magnetic field $B_{s}=55.3 \mathrm{~T}$ to be maintained along around $400 \mathrm{~m}$. To lower the required magnetic field strength, one may inject the electrons at a higher initial velocity, as can be seen from Eq. (4).

For electrons accelerated to tens of $\mathrm{GeV}$ energy or above, the question of whether radiation reaction effects play a role may arise. However, simulations based on the

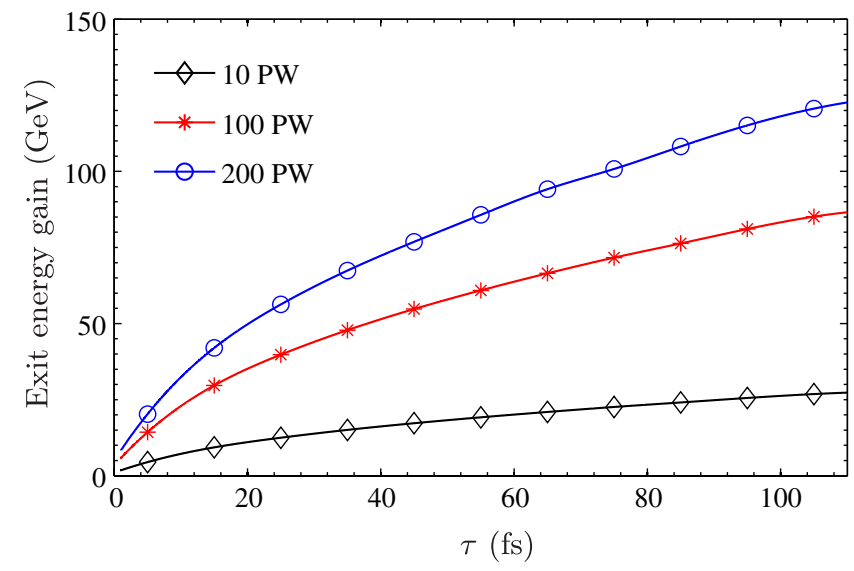

FIG. 6. Exit electron energy gain vs pulse duration for three laser powers. Optimal magnetic field strengths and waist radii at focus (obtained along the lines of Fig. 3, for every data point) have been used. The injection parameters are the same as in Fig. 3.
Landau-Lifshitz equation [37] have also been carried out which revealed that the electron dynamics is only marginally influenced by radiation reaction. This agrees well with the finding that, at intensities below $10^{18} \mathrm{~W} / \mathrm{cm}^{2}$, radiation reaction effects are negligible [38].

\section{SUMMARY AND CONCLUSIONS}

It has been demonstrated, in single- and many-particle simulations, that electrons may be accelerated to multi$\mathrm{GeV}$ energies, if launched into cyclotron autoresonance with a circularly polarized laser pulse, and employing parameters for the laser and required uniform magnetic field that are currently available, or under construction. Similar simulations have also been shown to lead to over $100-\mathrm{GeV}$ electron energy gains from envisaged laser pulses [25]. In all cases considered, the energy gradients exceed the known limits of conventional accelerators by at least 1 order of magnitude. Dedicated many-particle simulations reveal ultralow relative energy spreads $\Delta G / G$ of the order of $10^{-4}$ comparable with conventional accelerator and storage facilities [34] and suitable for high-precision particle physics experiments. However, we want to recall that the parameters used in this theoretical study particularly for the employed magnetic field strengths over long distances are out of the scope of near-future experiments.

\section{ACKNOWLEDGMENTS}

B. J. G. acknowledges discussions with T. V. Liseykina, A. Di Piazza, and M. Tamburini, B. J.G. and J.X. L. acknowledge hospitality at the American University of Sharjah (UAE) where part of this work was done, and Y.I.S. acknowledges support from the German Alexander von Humboldt Stiftung in Bonn. The work of Z. H. has been supported by the Alliance Program of the Helmholtz Association (HA216/EMMI).

[1] LHC-The Large Hadron Collider, http://hc.web.cern.ch/ lhe/.

[2] R. A. Snavely et al., Phys. Rev. Lett. 85, 2945 (2000).

[3] A.J. Mackinnon, M. Borghesi, S. Hatchett, M. Key, P. Patel, H. Campbell, A. Schiavi, R. Snavely, S. Wilks, and O. Willi, Phys. Rev. Lett. 86, 1769 (2001).

[4] S. Karsch, S. Düsterer, H. Schwoerer, F. Ewald, D. Habs, M. Hegelich, G. Pretzler, A. Pukhov, K. Witte, and R. Sauerbrey, Phys. Rev. Lett. 91, 015001 (2003).

[5] L. Romagnani et al., Phys. Rev. Lett. 95, 195001 (2005).

[6] B. M. Hegelich, B. J. Albright, J. Cobble, K. Flippo, S. Letzring, M. Paffett, H. Ruhl, J. Schreiber, R. K. Schulze, and J. C. Fernández, Nature (London) 439, 441 (2006).

[7] H. Schwoerer, S. Pfotenhauer, O. Jäckel, K.-U. Amthor, B. Liesfeld, W. Ziegler, R. Sauerbrey, K. W. D. Ledingham, and T. Esirkepov, Nature (London) 439, 445 (2006).

[8] L. Robson et al., Nat. Phys. 3, 58 (2006). 
[9] S.P.D. Mangles et al., Nature (London) 431, 535 (2004).

[10] C. G. R. Geddes, Cs. Toth, J. van Tilborg, E. Esarey, C. B. Schroeder, D. Bruhwiler, C. Nieter, J. Cary, and W. P. Leemans, Nature (London) 431, 538 (2004).

[11] J. Faure, Y. Glinec, A. Pukhov, S. Kiselev, S. Gordienko, E. Lefebvre, J.-P. Rousseau, F. Burgy, and V. Malka, Nature (London) 431, 541 (2004).

[12] J. Faure, C. Rechatin, A. Norlin, A. Lifschitz, Y. Glinec, and V. Malka, Nature (London) 444, 737 (2006).

[13] W. P. Leemans, B. Nagler, A. J. Gonsalves, Cs. Tóth, K. Nakamura, C. G. R. Geddes, E. Esarey, C. B. Schroeder, and S. M. Hooker, Nat. Phys. 2, 696 (2006).

[14] I. Blumenfeld et al., Nature (London) 445, 741 (2007).

[15] E. Esarey, C. B. Schroeder, and W. P. Leemans, Rev. Mod. Phys. 81, 1229 (2009).

[16] W. P. Leemans and E. Esarey, Phys. Today 62, 44 (2009).

[17] J. Singleton, C. H. Mielke, A. Migliori, G. S. Boebinger, and A. H. Lacerda, Physica (Amsterdam) 346B-347B, 614 (2004).

[18] See http://www.lanl.gov/orgs/mpa/nhmfl/ and http://www .lanl.gov/orgs/mpa/nhmfl/60TLP.shtml.

[19] J. Wosnitza, A. D. Bianchi, J. Freudenberger, J. Haase, T. Herrmannsdörfer, N. Kozlova, L. Schultz, Y. Skourski, S. Zherlitsyn, and S. A. Zvyagin, J. Magn. Magn. Mater. 310, 2728 (2007).

[20] H. J. Schneider-Muntau, A. V. Gavrilin, and C. A. Swenson, IEEE Trans. Appl. Supercond. 16, 926 (2006)

[21] M. D. Bird et al., IEEE Trans. Appl. Supercond. 19, 1612 (2009).

[22] A. Loeb and L. Friedland, Phys. Rev. A 33, 1828 (1986).
[23] Y. I. Salamin, F. H. M. Faisal, C. H. Keitel, Phys. Rev. A 62, 053809 (2000).

[24] J. L. Hirshfield and C. Wang, Phys. Rev. E 61, 7252 (2000).

[25] The Extreme Light Infrastructure, http://www.extremelight-infrastructure.eu.

[26] Y. I. Salamin, Appl. Phys. B 86, 319 (2007).

[27] M. Lax, W.H. Louisell, and W. B. McKnight, Phys. Rev. A 11, 1365 (1975).

[28] L. W. Davis, Phys. Rev. A 19, 1177 (1979).

[29] S. F. Martins, R. A. Fonseca, W. Lu, W. B. Mori, and L. O. Silva, Nat. Phys. 6, 311 (2010).

[30] G. H. Welsh, S. M. Wiggins, R. C. Issac, E. Brunetti, G. G. Manahan, M. R. Islam, S. Cipiccia, C. Aniculaesei, B. Ersfeld, and D. A. Jaroszynski, J. Plasma Phys. 78, 393 (2012).

[31] Y. I. Salamin, Z. Harman, and C. H. Keitel, Phys. Rev. Lett. 100, 155004 (2008).

[32] Z. Harman, Y. I. Salamin, B. J. Galow, and C. H. Keitel, Phys. Rev. A 84, 053814 (2011).

[33] Jian-Xing Li, Y. I. Salamin, B. J. Galow, and C. H. Keitel, Phys. Rev. A 85, 063832 (2012).

[34] S. Y. Lee, Accelerator Physics, 2nd Ed. (World Scientific, Singapore, 2004).

[35] C.L. O'Connel et al., Report No. SLAC-PUB-11195, 2005.

[36] B. J. Galow, Z. Harman, and C. H. Keitel, Opt. Express 18, 25950 (2010).

[37] L. D. Landau and E. M. Lifshitz, The Classical Theory of Fields (Elsevier, Oxford, 1975).

[38] A. Di Piazza, C. Müller, K.Z. Hatsagortsyan, and C. H. Keitel, Rev. Mod. Phys. 84, 1177 (2012). 